\title{
Rietveld analysis and maximum entropy method of powder diffraction for bundles of single-walled carbon nanotubes
}

\author{
Hiroaki Kadowaki, Akihito Nishiyama, Kazuyuki Matsuda, and Yutaka Maniwd \\ Department of Physics, Tokyo Metropolitan University, Hachioji-shi, Tokyo 192-0397, Japan \\ Shinzo Suzuki and Yohji Achiba \\ Department of Chemistry, Tokyo Metropolitan University, Hachioji-shi, Tokyo 192-0397, Japan \\ Hiromichi Kataura \\ Nanotechnology Research Institute, National Institute of Advanced \\ Industrial Science and Technology, Tsukuba, Ibaraki 305-8562, Japan
}

(Dated: November 20, 2018)

\begin{abstract}
The structure of bundles of single-walled carbon nanotubes (SWNT) has been refined by Rietveld analysis using neutron and X-ray powder diffraction data. Based on previous simulation studies of powder diffraction data of SWNT and standard Rietveld analyses, we have developed a pattern fit technique for SWNT which provides precise structure parameters. We also show that the present technique can be used with the maximum entropy method (MEM), which is complementary to the Rietveld analysis. Using the neutron diffraction data of pristine SWNT, we have successfully reconstructed the density of carbon nuclei and zero density in the inner cavity of SWNT by MEM.
\end{abstract}

PACS numbers:

\section{INTRODUCTION}

In recent years, adsorption of atoms and molecules in bundles of single-walled carbon nanotubes 1.2 (SWNT) is attracting interests for both fundamental and applied scientists,$\frac{3}{3}$ ranging from investigations of quasione-dimensional phases to applications of gas storage. 4 SWNTs are self-organized into a bundle with a two dimensional (2D) triangular lattice structure .5 In this bundle there are three main adsorption sites: an inner cavity of SWNT (tube or t), an interstitial channel (IC) between three SWNTs, a groove separating two SWNTs on the external surface of the bundle [see Fig. 11(a)] $\underline{3}^{3}$ For a small adsorbate, gas uptake may occur progressively on the three sites and finally covering the outside of the bundle as pressure is increased. Experimentally, gas uptakes can be studied by measuring adsorption isotherms. A more direct method of studying the adsorption sites, particularly useful for the IC and tube sites, is a diffraction experiment and its structure analysis of the $2 \mathrm{D}$ triangular lattice. Since the diffraction pattern is not affected by uptakes by impurities, such as graphite and nano-particles of graphite, the diffraction study provides complementary information to the adsorption isotherm.

Techniques of X-ray and neutron diffraction were used to demonstrate the triangular lattice of the bundles of SWNT,, 5 and to measure the diameter distribution of SWNT ${ }^{6.7}$ Powder diffraction patterns by bundles of SWNT with several adsorbates have been studied to determine the adsorption sites for $\mathrm{O}_{2}$ and $\mathrm{N}_{2},{ }^{8.9} \mathrm{CD}_{4}, 10$ Ar ${ }_{11}^{11} \mathrm{D}_{2}, 12$ and $\mathrm{I}_{n}, 13$ As pointed out in Ref. 8, intensity of the (10) reflection increases or decreases depending on adsorption at the IC or tube sites, respectively, being used as a simple experimental distinction.

When internal structures of adsorbed atoms or molecules in the tube site are studied, entire powder diffraction patterns have to be analyzed more carefully. Among a few examples of this kind, $\mathrm{C}_{60}$ and $\mathrm{H}_{2} \mathrm{O}$ molecules in SWNT were shown to form interesting onedimensional lattices in the inner cavity channel using X-ray powder diffraction. $6.14,15$ Adsorption of iodine in SWNT and its clustering in the tube and IC sites were studied using both X-ray and neutron powder diffraction 13 However the analyses of these powder diffraction patterns are in a primitive stage in comparison with recent elaborate techniques of the Rietveld analyses for three dimensional (3D) crystals ${ }^{16}$ The major difficulty for SWNT is brought about by randomness existing in bundles which is not controlled even by recent preparation methods. A bundle consists of different SWNTs with numerous chiralities, which are only characterized by a radius distribution with an average $R \sim 7 \AA$ and a width $\Delta R \sim 1 \AA$ [full width at half maximum (FWHM)]. In addition, radii of bundles in a sample vary in a typical range from $\sim 2 R$ to $\sim 20 R$. Small diameter bundles give rise to large broadening of Bragg peaks, which complicates the pattern fit method.

The first purpose of the present work is to extend the previous simulation-type studies of powder diffraction for SWNT 5 -6.7.13 to a quantitative Rietveld fit technique. We tried to provide a robust basis to this Rietveld analysis, which will facilitate to extract maximal information of the structure of adsorbates. In discussing this analysis in detail, it becomes clear that the powder diffraction patterns are more informative than previously expected. This leads us to another extension of the simulation studies, a possibility to exploit a Maximum entropy method $^{17.18}$ (MEM) for SWNT. Thus our second purpose is to formulate the MEM for SWNT, and to show its simplest application. This MEM can reconstruct the 
density of carbon and adsorbates at the tube site without assuming any structure models, which can be a complementary method to the Rietveld analysis, where a model structure is assumed from the beginning. In this paper, we restrict ourselves to methodologies and to analyzing powder diffraction data of pristine SWNT samples with no nominal adsorption, showing simple demonstrations of the Rietveld analysis and MEM.

\section{EXPERIMENTAL}

The raw soot of SWNT was prepared by laser vaporization ${ }^{\underline{5}}$ of a carbon rod including $\mathrm{Ni}$ and Co catalysts, and then purified as previously reported ${ }^{19}$ The average diameter of the SWNT used was $13.6 \AA$ close to $13.7 \AA$ of the $(10,10)$ SWNT. To remove adsorbed gases, the sample of SWNT was heated at $\sim 100^{\circ} \mathrm{C}$ in an evacuated aluminum container. A neutron diffraction experiment was performed using the triple-axis spectrometer 4G-GPTAS installed at JRR-3M JAERI (Tokai). The spectrometer was operated in a triple-axis configuration with collimations $30^{\prime}-80^{\prime}-80^{\prime}-$ open. Neutrons of the wave length $\lambda=2.44 \AA$ were selected by the pyrolytic-graphite (002) monochromator and analyzer. Higher-order neutrons were removed by the pyrolytic-graphite filter.

\section{STRUCTURE MODEL}

A simple structure mode ${ }^{5.6 .7}$ of a bundle of SWNTs for analyses of diffraction experiments is the $2 \mathrm{D}$ triangular lattice of cylinders which is shown in Fig. 1(a). In what follows, we use the terminology of neutron diffraction for clarity, which can be easily translated into that of X-ray diffraction. The cylinder represents the average nuclear density of carbon nuclei of SWNT in a bundle. The average density is regarded to have cylindrical symmetry, where atomic network structures of the graphene sheets are ignored, because SWNTs in the bundles are assumed to have various chiralities and to be self-organized without azimuthal correlation owing to the weak coupling by the van der Waals force. ${ }^{5}$

We assume that the nuclear density weighted by the scattering length of carbon nuclei $b_{\mathrm{C}}$ is well approximated by a Gaussian distribution with an average radius $R$ and a standard deviation $\sigma_{R}$ (FWHM is $\Delta R=\sqrt{8 \ln 2} \sigma_{R}$ ), which is illustrated in Fig. I(c). The density around the tube site $\rho_{\mathrm{t}}(r)=\rho_{\mathrm{C}}(r)$ is given by

$$
\rho_{\mathrm{C}}(r)=b_{\mathrm{C}} \sigma_{\mathrm{C}} \frac{1}{\sqrt{2 \pi} \sigma_{R}} \exp \left[-\frac{1}{2}\left(\frac{r-R}{\sigma_{R}}\right)^{2}\right],
$$

where $r$ and $\sigma_{\mathrm{C}}$ stand for the radius of the cylindrical coordinates and the surface density of carbon nuclei of the graphene sheet, respectively. This uniform cylinder model ( $\Delta R \rightarrow 0$ is usually assumed) on the triangular (a) bundle of SWNTs
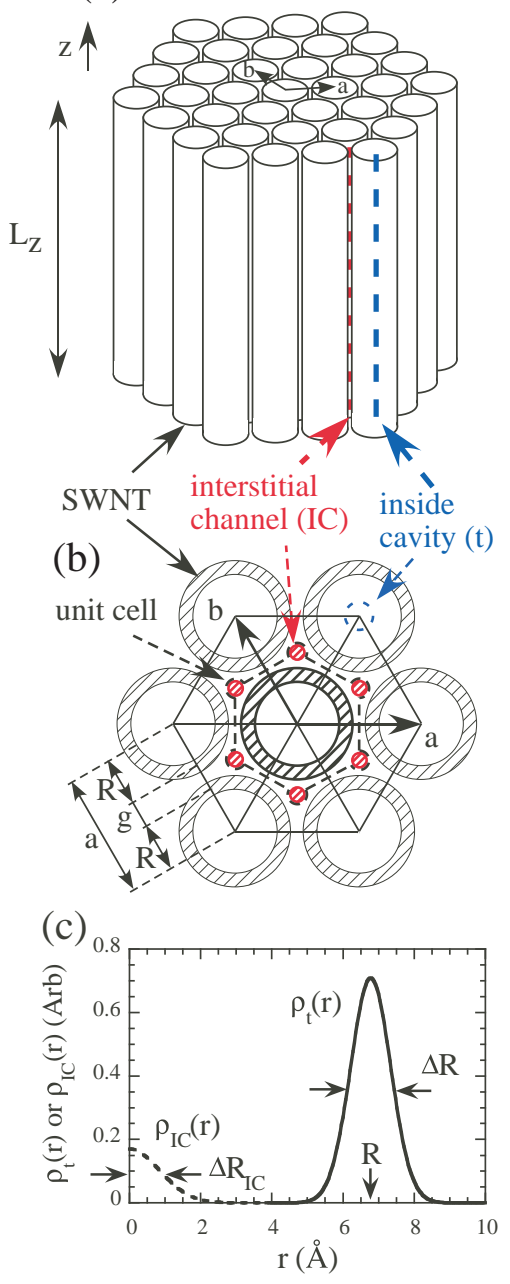

FIG. 1: (Color online) Structure model of a bundle of SWNTs for analyses of powder diffraction experiments. SWNTs are approximated by uniform cylinders, in which the network structure of the graphene sheet is neglected. They crystallize into the $2 \mathrm{D}$ triangular lattice as shown in (a), where the interstitial channel (IC) and the inside cavity of SWNT (t) are shown by dashed lines. The unit cell of the triangular lattice is the region surrounded by the dashed line in (b), where the density of the six ICs are multiplied by a weight factor of $\frac{1}{3}$ in calculating the structure factor. The nuclear densities weighted by scattering lengths of nuclei at the tube and IC sites, Eqs. (1) and (2) with parameters of the Rietveld fit result in Sec. IV are illustrated in (c), where $r$ represents radius from the tube or IC centers.

lattice was proposed to account for images of transmission electron microscope and X-ray powder diffraction patterns. ${ }^{\underline{5}}$ The fact that the atomic network can be neglected was justified by numerical calculations of powder diffraction patterns in a low wave-vector range of $Q \lesssim 2$ $\AA^{-1}$, which were performed for SWNT with single chirality $\underline{\underline{7}}$

The nano-scale channels in the bundle denoted by the tube (t) and IC sites are illustrated in Figs. 1(a) and 
(b). For calculations of diffraction patterns, adsorbed atoms in the tube site can be treated by an additional contribution to $\rho_{\mathrm{t}}(r)=\rho_{\mathrm{C}}(r)+\rho_{\text {ads }}(r)$. These atoms can show various structures and states in the wide inside space of SWNT, which should be studied by diffraction experiments ${ }^{6.14 .15}$ On the other hand, adsorbates in the IC site are localized in the narrow space surrounded by the three SWNTs. For the analysis of powder diffraction in the range $Q \lesssim 2 \AA^{-1}$, we can safely assume that the nuclear density is well approximated by a Gaussian function centered at the IC site with a small width $\sigma_{R_{\mathrm{IC}}}$ (half width at half maximum is $\Delta R_{\mathrm{IC}}=\sqrt{2 \ln 2} \sigma_{R_{\mathrm{IC}}}=1 \AA$ ). This nuclear density weighted by the scattering length of nuclei in the IC site $b_{\mathrm{IC}}$, which is shown in Fig. 1(c), is written by

$$
\rho_{\mathrm{IC}}(r)=b_{\mathrm{IC}} \rho_{\mathrm{IC}} \exp \left[-\frac{1}{2}\left(\frac{r}{\sigma_{R_{\mathrm{IC}}}}\right)^{2}\right],
$$

where $r$ and $\rho_{\mathrm{IC}}$ are the radius from the IC center and the peak nuclear density, respectively.

The unit cell of the 2D triangular lattice, which is used in calculating the structure factor, is depicted by the dashed line in Fig. 团(b). It should be noted that, to keep the six-fold symmetry of the structure factor as a function of wave vector, we choose a non-standard unit cell containing one tube site and six IC sites, the latter of which is weighted by $\frac{1}{3}$ for the structure factor calculation (cf. Eqs. A.6), A.7), and (A.8) in the appendix).

The average radius $R$ of SWNT in the bundle depends on its condition of the preparation, e.g. the local temperature of the furnace, implying that $R$ takes a number of values for bundles in a sample. Following Refs. 6 and $\mathbf{7}$, we assume that the probability density of $R$ is approximated by a Gaussian distribution with an average $R_{\text {av }}$ and a standard deviation $\sigma_{R_{\mathrm{av}}}$ (FWHM is $\left.\Delta R_{\mathrm{av}}=\sqrt{8 \ln 2} \sigma_{R_{\mathrm{av}}}\right)$, expressed by

$$
p(R)=\frac{1}{\sqrt{2 \pi} \sigma_{R_{\mathrm{av}}}} \exp \left[-\frac{1}{2}\left(\frac{R-R_{\mathrm{av}}}{\sigma_{R_{\mathrm{av}}}}\right)^{2}\right] .
$$

We note that the powder diffraction intensity by a bundle, which is calculated using a single value of $R$, has to be further averaged over the probability density. In this average, $R$ dependence of the intertube gap $g=a-2 R$ [see Fig. M(b)], where $a$ is the lattice constant of the triangular lattice, can be neglected $\underline{\underline{6}}$

\section{RIETVELD ANALYSIS}

In standard Rietveld analyses 16 of powder diffraction data of 3D crystals, the intensity of the diffracted beam at a scattering angle $2 \theta$ is fit to

$$
\begin{aligned}
I(2 \theta)= & s A(2 \theta) \sum_{K} m_{K}\left|F_{K}\right|^{2} L\left(2 \theta_{K}\right) \Phi\left(2 \theta-2 \theta_{K}\right) \\
& +y_{\mathrm{b}}(2 \theta),
\end{aligned}
$$

where $m_{K}, F_{K}$ and $L\left(2 \theta_{K}\right)=1 /\left(\sin \theta_{K} \sin 2 \theta_{K}\right)$ are the multiplicity, the structure factor, and the Lorentz factor, respectively, of the $K$-th reflection with the scattering angle $2 \theta_{K} ; s, A(2 \theta)$ and $y_{\mathrm{b}}(2 \theta)$ represent the scale factor, the absorption factor, and the background, respectively. The profile function $\Phi\left(2 \theta-2 \theta_{K}\right)$, which is normalized by $\int \Phi\left(2 \theta-2 \theta_{K}\right) d(2 \theta)=1$, shows $K$-dependent broadening due to the instrumental resolution and the sample crystallinity. The functional form of $\Phi$ is often approximated by the pseudo-Voigt function, which is further modified to an asymmetric form $: \underline{16.20}$

For a bundle of SWNTs, the scattering cross-section due to the structure model in the previous section, which is derived in the appendix [cf. Eq. A.11] ], is given by

$$
\begin{aligned}
\frac{d \sigma}{d \Omega}(\boldsymbol{Q}) \simeq & L_{z} N_{\mathrm{t}} \frac{16 \pi^{3}}{\sqrt{3} a^{2}} \sum_{\boldsymbol{G}} \delta\left(Q_{z}\right) \hat{\delta}\left(\boldsymbol{Q}_{\perp}-\boldsymbol{G}\right) \\
& \times\left|F\left(\boldsymbol{Q}_{\perp}\right)\right|^{2},
\end{aligned}
$$

where $L_{z}$ and $N_{\mathrm{t}}$ stand for the length of SWNT [see Fig. [(a)] and the number of SWNTs in the bundle, respectively. The wave vector $\boldsymbol{Q}=Q_{z} \boldsymbol{e}_{z}+\boldsymbol{Q}_{\perp}$ is decomposed into the $z$ component $Q_{z}$ parallel to the direction along SWNT, and its perpendicular components $\boldsymbol{Q}_{\perp}=h \boldsymbol{a}^{*}+k \boldsymbol{b}^{*}$. The structure factor $F\left(\boldsymbol{Q}_{\perp}\right)$ is defined by Eq. A.4 $)$, and the function $\hat{\delta}\left(\boldsymbol{Q}_{\perp}-\boldsymbol{G}\right)$ is the broadened 2D $\delta$ function peaked at a reciprocal lattice point $\boldsymbol{G}$ of the triangular lattice. Simulations of powder diffraction by bundles of SWNTs have been performed to study $\mathrm{X}$-ray and neutron diffraction data $\frac{5,6,7.8,10,11,12,13.14,15,21}{1}$

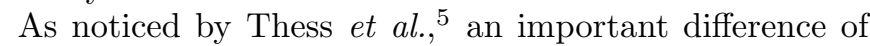
Eq. (5) from that of the 3D crystals is the broadening of the $2 \mathrm{D} \delta$ function by the finite size effect of the bundle radius. This broadening of the Bragg peaks is much larger than instrumental resolutions of powder diffractometers.

Powder averaged intensity of Eq. (5), corresponding to the first term of Eq. (4), is given by [cf. Eq. (A.14)]

$$
\begin{aligned}
\tilde{I}(Q) \simeq & s A(Q) \sum_{K} m_{K}\left|F\left(Q \hat{\boldsymbol{G}}_{K}\right)\right|^{2} L(Q) \\
& \times \phi\left(Q-\left|\boldsymbol{G}_{K}\right|\right),
\end{aligned}
$$

where $F\left(Q \hat{\boldsymbol{G}}_{K}\right)$ is an approximation of the powder averaged structure factor [cf. Eqs. A.11, A.12, and [A.13] ], and $\phi\left(Q-\left|\boldsymbol{G}_{K}\right|\right)$ is the normalized profile function, which is further modified to an asymmetric form ${ }^{16.20}$ The use the wave vector $Q=|Q|$ instead of scattering angle $2 \theta$ is the previous convention, 5.7 .8 where the Lorentz factor becomes $L(Q)=1 / \sin ^{2} \theta$ or $\propto 1 / Q^{2}$. By further taking the average of Eq. (6) over the distribution of the radius $R$ of SWNT, Eq. (3), we arrive at an expression

$$
\begin{aligned}
I(Q) & =\langle\tilde{I}(Q)\rangle_{R}+y_{\mathrm{b}}(Q) \\
\langle\tilde{I}(Q)\rangle_{R} & =\int_{0}^{\infty} p(R) \tilde{I}(Q) d R
\end{aligned}
$$

which is used as the calculated intensity for the present Rietveld fit. 
Neutron powder diffraction pattern of pristine SWNT with no nominal adsorbates measured at $300 \mathrm{~K}$ is shown in Fig. 2] The instrumental resolution with the present condition is not negligibly small compared to the broadening by the finite size effect. This additional resolution correction was performed by convoluting $\langle\tilde{I}(Q)\rangle_{R}$ of Eq. (7) with the Gaussian resolution function. ${ }^{22}$ The profile function $\phi\left(Q-\left|\boldsymbol{G}_{K}\right|\right)$ of Eq. (6) was determined by the similar Rietveld fit based on Eq. (7) using the previous X-ray diffraction data ${ }^{6}$ of pristine SWNT. These data were measured using a synchrotron X-ray powder diffractometer with far higher $Q$ resolution, where no resolution convolution was made. The profile function could be well approximated by superposition of four Gaussian functions. The resulting Rietveld analysis is plotted in the inset of Fig. 2 Using this profile function, the Rietveld analysis of the neutron powder diffraction was performed. The background $y_{\mathrm{b}}(Q)$ of Eq. (7) was chosen to be a polynomial in $Q^{-1}, \sum_{n=0}^{3} c_{n} Q^{-n}$, with additional three Gaussian functions centered around $Q \sim 1.8 \AA^{-1}$, where impurity peaks by graphite and nano-particles of graphite exist. The fitted curve shown in Fig. 2 is in good agreement with the observed data.

The fitted parameters of the model structure are: $R_{\mathrm{av}}=6.78 \pm 0.02 \AA, \Delta R_{\mathrm{av}}=0.56 \pm 0.04 \AA$ (FWHM), $\Delta R=1.32 \pm 0.08 \AA$ (FWHM), $g=3.34 \pm 0.04 \AA$, $\Delta R_{\mathrm{IC}}=1 \AA$ (fixed), and $b_{\mathrm{IC}} \rho_{\mathrm{IC}} /\left(b_{\mathrm{C}} \sigma_{\mathrm{C}}\right)=0.21 \pm 0.08$ $\AA^{-1}$, for the neutron diffraction data. Those for the X-ray diffraction data are: $R_{\text {av }}=6.715 \pm 0.005 \AA$, $\Delta R_{\mathrm{av}}=0.68 \pm 0.01 \AA$ (FWHM), $\Delta R=1.26 \pm 0.03$ $\AA$ (FWHM), $g=3.23 \pm 0.01 \AA, \Delta R_{\mathrm{IC}}=1 \AA$ (fixed), and $\rho_{\mathrm{e}, \mathrm{IC}} / \sigma_{\mathrm{e}, \mathrm{C}}=0.11 \pm 0.04 \AA^{-1}$, where $\rho_{\mathrm{e}, \mathrm{IC}}$ and $\sigma_{\mathrm{e}, \mathrm{C}}$ are the peak electron density at the IC site and the surface electron density of the graphene sheet, respectively. These parameters of the X-ray data are consistent with the previous work ${ }^{6}$ They also agree reasonably well with the parameters of the neutron data, considering the slight sample difference. We conclude that the Rietveld fittings have been successfully performed for the neutron and Xray powder diffraction data.

\section{MAXIMUM ENTROPY METHOD}

The structural information of the unit cell is contained in the structure factor $F(\boldsymbol{Q})$. In a Rietveld analysis of an ordinary $3 \mathrm{D}$ crystal, structure factors are observed at a number of discrete wave vectors $\boldsymbol{G}$. On the other hand, there are only 10 reflections in the present neutron powder diffraction data of SWNT (see Fig. 2). From this small number, one may think that it is very difficult to determine many structural parameters. However, this is not simply the case, owing to the other aspect of the important difference, i.e., the large width of the profile function, showing substantial overlaps with neighboring reflections, which is easily seen in the range $0.4<Q<0.8$ $\AA^{-1}$ of Fig. 2. This overlap suggests a possibility to make use of the structure factor in a continuous $Q$ range. If the

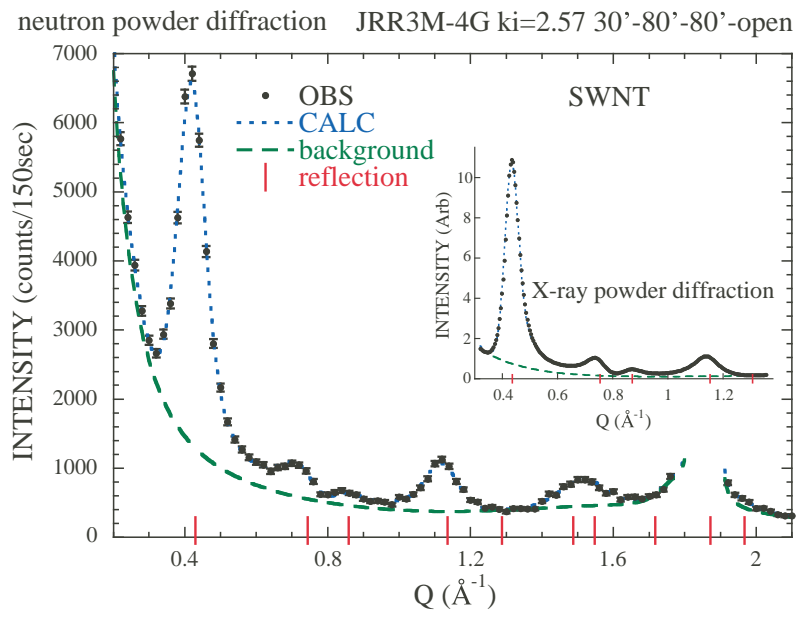

FIG. 2: (Color online) Neutron diffraction pattern of SWNTs at $300 \mathrm{~K}$. Observed and calculated intensities are plotted by closed circle and dotted lines, respectively. Background is plotted by dashed line. Vertical bars stand for Bragg peak positions with $a=2 R_{\mathrm{av}}+g$. X-ray powder diffraction of the previous work ${ }^{6}$ and its Rietveld analysis are shown in the inset.

observer range of $0.2<Q<2.1 \AA^{-1}$ is fully available, one may think that the powder diffraction data contain more information than the simple expectation. As noticed in Ref. 21, this is the reason why the parameter $R_{\mathrm{av}}$ is precisely determined by powder diffraction of SWNT. In the following, we discuss a method which takes advantage of the availability of the continuous $Q$ range.

Adsorbates in the IC site, which are localized in the small space, cannot have internal structures except for those in the direction along SWNT. Thus we assume that the IC part of the structure factor has only one parameter $\rho_{\text {IC }}$, which can be easily determined. Then remaining problem is the structure at the tube site, SWNT and its inside, which is expressed by $\rho_{\mathrm{t}}(r)$. The density $\rho_{\mathrm{t}}(r)$ is related to the tube part of the structure factor by [cf. Eq. A.9.

$$
F_{\mathrm{t}}(Q)=2 \pi \int_{0}^{\infty} r J_{0}(Q r) \rho_{\mathrm{t}}(r) d r,
$$

where $J_{0}(Q r)$ is the cylindrical Bessel function. Thus the problem is reduced to how to determine $\rho_{\mathrm{t}}(r)$ from $F_{\mathrm{t}}(Q)$. Since Eq. (8) is the Fourier-Bessel transform, a solution is to use the inverse transform

$$
\rho_{\mathrm{t}}(r)=\frac{1}{2 \pi} \int_{0}^{\infty} Q J_{0}(Q r) F_{\mathrm{t}}(Q) d Q .
$$

However an application of this inversion to the neutron diffraction data failed, because the inverted $\rho_{\mathrm{t}}(r)$ showed a spurious oscillation, which is a cut-off effect due to insufficient $Q$ range. A well known method to avoid this cut-off effect of Fourier transform is MEM,, 17.23 which is being successfully used in single-crystal and powder diffraction analyses. $16,18,24$ 
In general, MEM is based on a linear relation between observed and calculated quantities (data and image in the MEM terminology 23 ). This relation is Eq. (8) for $F_{\mathrm{t}}(Q)$ (data) and $\rho_{\mathrm{t}}(r)$ (image). To apply a general purpose MEM algorithm, 23 a discrete version of the linear relation

$$
F_{\mathrm{t}}\left(Q_{n}\right)=2 \pi \sum_{m} J_{0}\left(Q_{n} r_{m}\right) r_{m} \Delta r_{m} \rho_{\mathrm{t}}\left(r_{m}\right)
$$

was used as a basis for the present MEM. The first step of the MEM is to determine observed values of $F_{\mathrm{t}}\left(Q_{n}\right)$. We calculated these by a Rietveld fit, where $F_{\mathrm{t}}\left(Q_{n}\right)$ are fitting parameters and intermediate $F_{\mathrm{t}}(Q)$ is evaluated by a linear interpolation. In this fitting the other parameters: the background parameters of $y_{\mathrm{b}}(Q), s, b_{\mathrm{IC}} \rho_{\mathrm{IC}}$, $\Delta R_{\mathrm{IC}}, g, R_{\mathrm{av}}$, and $\Delta R_{\mathrm{av}}$, were fixed to the values of the previous section. It should be noted that the three parameters, $g, R_{\mathrm{av}}$, and $\Delta R_{\mathrm{av}}$, and the SWNT radius $R$ still have meaning in connection with the lattice constant $a=2 R+g$. Thus the fitting parameters $F_{\mathrm{t}}\left(Q_{n}\right)$ depend on $R$, and we refer to this $R$ dependence explicitly by $\left[F_{\mathrm{t}}\left(Q_{n}\right)\right]_{R}$. In performing the average over $R$ [cf. Eq. (77)], we assumed $R$ dependence

$$
\left[F_{\mathrm{t}}\left(Q_{n}\right)\right]_{R}=\frac{R}{R_{\mathrm{av}}} F_{\mathrm{t}}^{(0)}\left(\frac{R}{R_{\mathrm{av}}} Q_{n}\right),
$$

which is a good approximation for empty SWNT. The Rietveld fit was carried out and resulting parameters $\left[F_{\mathrm{t}}\left(Q_{n}\right)\right]_{R=R_{\mathrm{av}}}$ are shown in Fig. 3(a) together with that of the Rietveld analysis in the previous section.

The second step of the present MEM is to reconstruct $\rho_{\mathrm{t}}\left(r_{m}\right)$ from $\left[F_{\mathrm{t}}\left(Q_{n}\right)\right]_{R=R_{\mathrm{av}}}$. We carried out this using the general purpose MEM algorithm ${ }^{23}$ The reconstructed $\rho_{\mathrm{t}}\left(r_{m}\right)$ is plotted in Fig. B(b), where the Gaussian function Eq. (II) with $R=R_{\mathrm{av}}$ determined by the Rietveld analysis in the previous section is also shown. One can see that the Rietveld and MEM results agree surprisingly well. The structure factors $\left[F_{\mathrm{t}}\left(Q_{n}\right)\right]_{R=R_{\text {av }}}$ were calculated using the MEM reconstructed $\rho_{\mathrm{t}}\left(r_{m}\right)$ and Eq. (10), and are plotted in Fig. 3(a). They agree well with the observed $\left[F_{\mathrm{t}}\left(Q_{n}\right)\right]_{R=R_{\mathrm{av}}}$ apart from small discrepancy around $Q \sim 0.55 \AA^{-1}$, implying that the present MEM gives self-consistent results. Therefore we conclude that the continuous $Q$ data of structure factor have been excellently analyzed by the present MEM formulation.

\section{DISCUSSION}

The advantage of MEM ${ }^{17.23}$ is providing a way to choose a maximally non-committal nuclear density without any structure models among a series of nuclear densities consistent with experimental data. Thus MEM is a complementary method to the Rietveld analysis, in which an empirical structure model is assumed from the beginning. Since the formulation of MEM discussed in
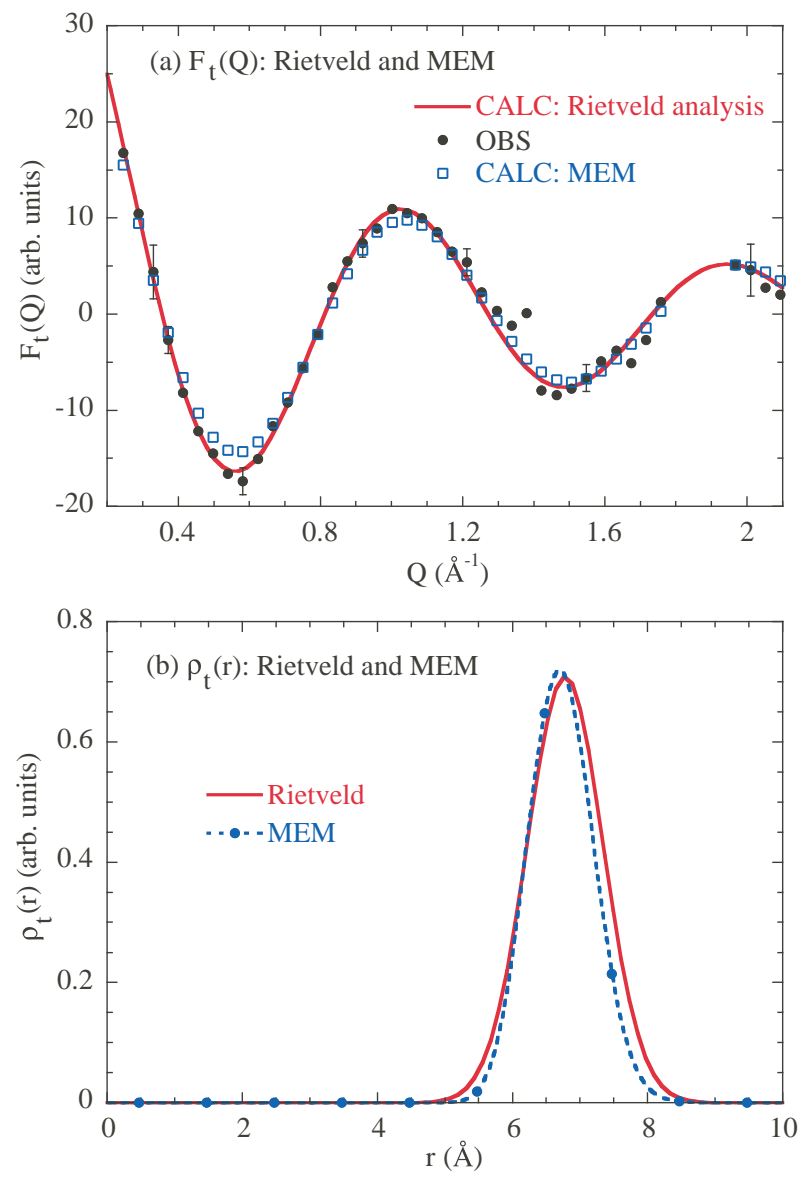

FIG. 3: (Color online) (a) Structure factors of the tube site $F_{\mathrm{t}}(Q)$ with $R=R_{\mathrm{av}}$ are plotted as a function of wave vector. The full line is the result of the Rietveld analysis in Sec. IV] Closed circle represents the observed $\left[F_{\mathrm{t}}\left(Q_{n}\right)\right]_{R=R_{\mathrm{av}}}$ determined by the fit described in the text. Open square is the calculated $\left[F_{\mathrm{t}}\left(Q_{n}\right)\right]_{R=R_{\text {av }}}$ using Eq. (10) and $\rho_{\mathrm{t}}\left(r_{m}\right)$ obtained by MEM. (b) Nuclear densities at the tube site obtained by the Rietveld analysis in Sec. IV with $R=R_{\mathrm{av}}$ and MEM.

Sec. $\mathbb{\nabla}$ partly relies on the result of the Rietveld analysis of Sec. IV] we have to check consistency between the MEM reconstructed nuclear density and that of the empirical structure model. Fig. 31 b) definitely warrants this consistency for the pristine SWNT sample. For general cases, where atoms are adsorbed inside SWNT, the consistency is not necessarily obtained after the first trial of MEM. If the first consistency check is negative, we have to adjust the structure model considering the first MEM reconstructed nuclear density and perform second Rietveld and MEM analyses. Depending on the second consistency check, we may arrive at a final answer or continue this iterative procedure. This kind of iterative use of the Rietveld and MEM analyses have been skillfully used for powder diffraction of 3D crystals 16.24 Applications of the present Rietveld and MEM analyses to SWNT with adsorbates in the tube site are awaited. 


\section{CONCLUSIONS}

We have developed a pattern fit technique, Rietveld analysis, for powder diffraction data of bundles of SWNTs, by extending previous simulation studies. Neutron and X-ray powder diffraction data of pristine SWNT samples have been successfully analyzed by this Rietveld analysis. The high fitting quality indicates that the analysis will be useful to obtain structure parameters of adsorbates in SWNT samples. We also have exploited a MEM for reconstructing nuclear or electron density at the tube site with the use of the pattern fit technique. We have applied this MEM to the neutron diffraction data and reconstructed the nuclear density of SWNT consistent with the Rietveld result.

\section{Acknowledgments}

We are grateful to K. Shibata and H. Yoshizawa for valuable discussions. H. Kataura acknowledges a support by Industrial Technology Research Grant Program in '03 from New Energy and Industrial Technology Development Organization (NEDO) of Japan.

\section{APPENDIX: SCATTERING CROSS-SECTION}

The neutron scattering cross-section by a bundle of SWNTs is calculated using the general formula ${ }^{25}$

$$
\frac{d \sigma}{d \Omega}(\boldsymbol{Q})=\left|\int \exp (i \boldsymbol{Q} \cdot \boldsymbol{r}) \rho(\boldsymbol{r}) d \boldsymbol{r}\right|^{2},
$$

where $\rho(\boldsymbol{r})$ stands for the nuclear density weighted by the scattering length of each nucleus. On the basis of this equation one can discuss the Bragg scattering, scattering from a single SWNT, cluster of atoms or molecules, small angle scattering, etc.

For the Bragg scattering due to the model structure shown in Fig. 11 the nuclear density is expressed by

$$
\begin{aligned}
\rho\left(\boldsymbol{r}=\boldsymbol{r}_{\perp}+z \boldsymbol{e}_{z}\right)= & \sum_{\boldsymbol{r}_{\perp, \mathrm{t}}} \rho_{\mathrm{t}}\left(\left|\boldsymbol{r}_{\perp}-\boldsymbol{r}_{\perp, \mathrm{t}}\right|\right) \\
& +\sum_{\boldsymbol{r}_{\perp, \mathrm{IC}}} \rho_{\mathrm{IC}}\left(\left|\boldsymbol{r}_{\perp}-\boldsymbol{r}_{\perp, \mathrm{IC}}\right|\right) .
\end{aligned}
$$

The first term is the nuclear density of SWNT and adsorbates at the tube site $\boldsymbol{r}_{\perp, \mathrm{t}}=n_{a} \boldsymbol{a}+n_{b} \boldsymbol{b}$. The second term is the nuclear density of adsorbates at the IC site $\boldsymbol{r}_{\perp \text {,IC }}=$ $\boldsymbol{r}_{\perp, \mathrm{t}} \pm\left(\frac{1}{3} \boldsymbol{a}+\frac{2}{3} \boldsymbol{b}\right)\left[\right.$ or $\left.\pm\left(\frac{2}{3} \boldsymbol{a}+\frac{1}{3} \boldsymbol{b}\right), \pm\left(\frac{1}{3} \boldsymbol{a}-\frac{1}{3} \boldsymbol{b}\right)\right]$. By inserting Eq. A.2 in Eq. A.1 and performing the integration along the $z$ axis in $0<z<L_{z}$ with large $L_{z} \geq 1$ $\mu \mathrm{m}, \frac{5}{,}$ one has

$$
\begin{aligned}
\frac{d \sigma}{d \Omega}(\boldsymbol{Q})= & 2 \pi L_{z} \delta\left(Q_{z}\right)\left|\sum_{\boldsymbol{r}_{\perp, \mathrm{t}}} \exp \left(i \boldsymbol{Q}_{\perp} \cdot \boldsymbol{r}_{\perp, \mathrm{t}}\right)\right|^{2} \\
& \times\left|F\left(\boldsymbol{Q}_{\perp}\right)\right|^{2}
\end{aligned}
$$

where the summation is taken over $2 \mathrm{D}$ triangular lattice positions. The structure factor $F\left(\boldsymbol{Q}_{\perp}\right)$ is given by

$$
F\left(\boldsymbol{Q}_{\perp}\right)=\int_{\text {unit cell }} \exp \left(i \boldsymbol{Q}_{\perp} \cdot \boldsymbol{r}_{\perp}\right) \rho\left(\boldsymbol{r}_{\perp}\right) d \boldsymbol{r}_{\perp},
$$

where the unit cell is shown in Fig. 1(b). We note that although the $\delta$ function in Eq. A.3 is sharp along the $Q_{z}$ direction, the lattice sum peaks at reciprocal lattice points $\boldsymbol{G}$ with a finite width in the $\boldsymbol{Q}_{\perp}$ space. ${ }^{5}$ The latter would become $2 \mathrm{D} \delta$ function only in the limit of the infinitely large radius of the bundle, as

$$
\left|\sum_{\boldsymbol{r}_{\perp, \mathrm{t}}} \exp \left(i \boldsymbol{Q}_{\perp} \cdot \boldsymbol{r}_{\perp, \mathrm{t}}\right)\right|^{2} \rightarrow N_{\mathrm{t}} \frac{8 \pi^{2}}{\sqrt{3} a^{2}} \sum_{\boldsymbol{G}} \delta\left(\boldsymbol{Q}_{\perp}-\boldsymbol{G}\right)
$$

where $N_{\mathrm{t}}(\rightarrow \infty)$ is the number of SWNTs.

The finite width of the Bragg-like scattering in Eq. A.3 by small $N_{\mathrm{t}}$ requires us to carefully treat the structure factor of Eq. A.4, because $F\left(\boldsymbol{Q}_{\perp}\right)$ should be evaluated for continuously varying $\boldsymbol{Q}_{\perp}$. In view of the rope shape of TEM images of bundles,,$^{\frac{5}{-}}$ which has quasi-six-fold symmetry, it is probably adequate to keep the six-fold symmetry of $F\left(\boldsymbol{Q}_{\perp}\right)$ for continuously vary$\operatorname{ing} Q_{\perp}$. It can be retained for the unit cell shown in Fig. 1(b), explicitly using the nuclear density

$$
\rho\left(\boldsymbol{r}_{\perp}\right)=\rho_{\mathrm{t}}\left(\left|\boldsymbol{r}_{\perp}\right|\right)+\frac{1}{3} \sum_{i=1}^{6} \rho_{\mathrm{IC}}\left(\left|\boldsymbol{r}_{\perp}-\boldsymbol{r}_{\perp, \mathrm{IC}, i}\right|\right)
$$

in Eq. A.4, where $\boldsymbol{r}_{\perp, \mathrm{IC}, i}$ is the surrounding six IC sites around the tube site $\boldsymbol{r}_{\perp, t}=0$. Substituting Eq. A.6 for Eq. A.4, the structure factor becomes

$$
F\left(\boldsymbol{Q}_{\perp}\right)=F_{\mathrm{t}}\left(\left|\boldsymbol{Q}_{\perp}\right|\right)+g\left(\boldsymbol{Q}_{\perp}\right) F_{\mathrm{IC}}\left(\left|\boldsymbol{Q}_{\perp}\right|\right),
$$

where

$$
\begin{aligned}
g\left(\boldsymbol{Q}_{\perp}\right. & \left.=h \boldsymbol{a}^{*}+k \boldsymbol{b}^{*}\right)=\frac{2}{3}\left\{\cos \left[\frac{2 \pi}{3}(2 h+k)\right]\right. \\
& \left.+\cos \left[\frac{2 \pi}{3}(h+2 k)\right]+\cos \left[\frac{2 \pi}{3}(h-k)\right]\right\} .
\end{aligned}
$$

The two terms in Eq. A.7 are the contribution from the tube site

$$
\begin{aligned}
F_{\mathrm{t}}\left(\left|\boldsymbol{Q}_{\perp}\right|\right) & =\int \exp \left(i \boldsymbol{Q}_{\perp} \cdot \boldsymbol{r}_{\perp}\right) \rho_{\mathrm{t}}\left(\left|\boldsymbol{r}_{\perp}\right|\right) d \boldsymbol{r}_{\perp} \\
& =2 \pi \int_{0}^{\infty} r J_{0}\left(Q_{\perp} r\right) \rho_{\mathrm{t}}(r) d r
\end{aligned}
$$

and that from the IC site

$$
\begin{aligned}
F_{\mathrm{IC}}\left(\left|\boldsymbol{Q}_{\perp}\right|\right) & =\int \exp \left(i \boldsymbol{Q}_{\perp} \cdot \boldsymbol{r}_{\perp}\right) \rho_{\mathrm{IC}}\left(\left|\boldsymbol{r}_{\perp}\right|\right) d \boldsymbol{r}_{\perp} \\
& =2 \pi \int_{0}^{\infty} r J_{0}\left(Q_{\perp} r\right) \rho_{\mathrm{IC}}(r) d r .
\end{aligned}
$$


Considering Eqs. A.3 and A.5, the neutron scattering cross-section due to the Bragg-like scattering of the $2 \mathrm{D}$ triangular lattice is summarized as

$$
\begin{aligned}
\frac{d \sigma}{d \Omega}(\boldsymbol{Q}) \simeq & L_{z} N_{\mathrm{t}} \frac{16 \pi^{3}}{\sqrt{3} a^{2}} \sum_{\boldsymbol{G}} \delta\left(Q_{z}\right) \hat{\delta}\left(\boldsymbol{Q}_{\perp}-\boldsymbol{G}\right) \\
& \times\left|F\left(\boldsymbol{Q}_{\perp}\right)\right|^{2}
\end{aligned}
$$

where the function $\hat{\delta}\left(\boldsymbol{Q}_{\perp}-\boldsymbol{G}\right)$ is a broadened 2D $\delta$ function normalized by $\int \hat{\delta}\left(\boldsymbol{Q}_{\perp}-\boldsymbol{G}\right) d \boldsymbol{Q}_{\perp}=1$.

To calculate intensity of powder diffraction, this crosssection is further averaged over all orientation of the bundle, or equivalently over all direction of $\boldsymbol{Q} \stackrel{13}{\stackrel{13}{1} \text { i.e., }}$

$$
\begin{aligned}
\tilde{I}(Q) & =\left\langle\frac{d \sigma}{d \Omega}(\boldsymbol{Q})\right\rangle_{|\boldsymbol{Q}|=Q, \text { powder av }} \\
& =\frac{1}{4 \pi Q^{2}} \int \frac{d \sigma}{d \Omega}(\boldsymbol{Q}) d \boldsymbol{S}_{\boldsymbol{Q}},
\end{aligned}
$$

where $d \boldsymbol{S}_{\boldsymbol{Q}}$ is the infinitesimal area on the sphere of radius $Q$. Since the numerical evaluation of this powder average took too long time to perform the Rietveld fit- ting, we made an ad hoc approximation given by

$$
\begin{aligned}
\tilde{I}(Q) \simeq & L_{z} N_{\mathrm{t}} \frac{16 \pi^{3}}{\sqrt{3} a^{2}} \sum_{\boldsymbol{G}} \phi(Q-|\boldsymbol{G}|) \frac{1}{4 \pi Q^{2}} \\
& \times|F(Q \hat{\boldsymbol{G}})|^{2}
\end{aligned}
$$

where a profile function $\phi(Q-G)$ normalized by $\int \phi(Q-$ $G) d Q=1$ is used, and $\hat{\boldsymbol{G}}=\boldsymbol{G} / G$. We checked numerically that Eq. A.13 is a good approximation for the fits of the present experimental data. By using the sixfold symmetry of the structure factor, we finally write Eq. A.13) in a form appropriate for the powder diffraction

$$
\begin{aligned}
\tilde{I}(Q) \simeq & L_{z} N_{\mathrm{t}} \frac{4 \pi^{2}}{\sqrt{3} a^{2}} \sum_{K} m_{K} \phi\left(Q-\left|\boldsymbol{G}_{K}\right|\right) \frac{1}{Q^{2}} \\
& \times\left|F\left(Q \hat{\boldsymbol{G}}_{K}\right)\right|^{2},
\end{aligned}
$$

where $m_{K}$ is the multiplicity.
* Also at CREST, JST (Japan Science and Technology Corporation).

1 S. Iijima and T. Ichihashi, Nature (London) 363, 603 (1993).

2 D. S. Bethune, C. H. Kiang, M. S. de Vries, G. Gorman, R. Savoy, J. Vasquez, and R. Beyers, Nature (London) 363, 605 (1993).

3 M. M. Calbi, M. W. Cole, S. M. Gatica, M. J. Bojan, and G. Stan, Rev. Mod. Phys. 73, 857 (2001).

4 M. R. Pederson and J. Q. Broughton, Phys. Rev. Lett. 69, 2689 (1992).

5 A. Thess, R. Lee, P. Nikolaev, H. Dai, P. Petit, J. Robert, C. Xu, Y. H. Lee, S. G. Kim, A. G. Rinzler, D. T. Colbert, G. E. Scuseria, D. Tomanek, J. E. Fischer, and R. E. Smally, Science 273, 483 (1996).

6 M. Abe, H. Kataura, H. Kira, T. Kodama, S. Suzuki, Y. Achiba, K. Kato, M. Takata, A. Fujiwara, K. Matsuda, and Y. Maniwa, Phys. Rev. B 68, 041405(R) (2003).

7 S. Rols, R. Almairac, L. Henrard, E. Anglaret, and J.-L. Sauvajol, Eur. Phys. J. B 10, 263 (1999).

8 Y. Maniwa, Y. Kumazawa, Y. Saito, H. Tou, H. Kataura, H. Ishii, S. Suzuki, Y. Achiba, A. Fujiwara, and H. Suematsu, Jpn. J. Appl. Phys. 38, L668 (1999).

9 A. Fujiwara, K. Ishii, H. Suematsu, H. Kataura, Y. Maniwa, S. Suzuki, and Y. Achiba, Chem. Phys. Lett. 336, 205 (2001).

10 M. Muris, M. Bienfait, P. Zeppenfeld, N. Dupont-Pavlosky, M. Johnson, O. E. Vilches, and T. Wilson, Appl. Phys. A 74, S1293 (2002).

11 M. Bienfait, P. Zeppenfeld, N. Dupont-Pavlovsky, J.-P. Palmari, M. R. Johnson, T. Wilson, M. DePies, and O. E. Vilches, Phys. Rev. Lett. 91, 35503 (2003).

12 S. Challet, P.Azaïs, R. J.-M. Pellenq, and L. Duclaux,
Chem. Phys. Lett. 377, 544 (2003).

13 N. Bendiab, R. Almairac, S. Rols, R. Aznar, J.-L. Sauvajol, and I. Mirebeau, Phys. Rev. B 69, 195415 (2004).

14 Y. Maniwa, H. Kataura, M. Abe, S. Suzuki,Y. Achiba, H. Kira, and K. Matsuda, J. Phys. Soc. Jpn. 71, 2863 (2002).

15 Y. Maniwa, H. Kataura, M. Abe, A. Udaka, S. Suzuki,Y. Achiba, H. Kira, K. Matsuda, H. Kadowaki, and Y. Okabe, Chem. Phys. Lett. 401, 534 (2005).

16 e.g. F. Izumi, Chap. 13 in The Rietveld Method, edited by R. A. Young (Oxford Univ. Press, 1993) and documentation of RIETAN-2000 available at http://homepage.mac.com/fujioizumi/

17 S. F. Gull and G. J. Daniell, Nature (London) 272, 686 (1978).

18 D. M. Collins, Nature (London) 298, 49 (1982).

19 H. Kataura, Y. Maniwa, T. Kodama, K. Kikuchi, H. Hirahara, K. Suenaga, S. Iijima, S. Suzuki, Y. Achiba, and W. Kraetschmer, Synth. Met. 121, 1195 (2001).

20 C. J. Howard: J. Appl. Crystallogr. 15, 615 (1982).

21 Y. Maniwa, R. Fujiwara, H. Kira, H. Tou, H. Kataura, S. Suzuki, Y. Achiba, E. Nishibori, M. Takata, M. Sakata, A. Fujiwara, and H. Suematsu, Phys. Rev. B 64, 241402(R) (2001).

22 M. J. Cooper and R. Nathans, Acta Cryst. 23, 357 (1967).

23 J. Skilling and R. K. Bryan, Mon. Not. R. Astron. Soc. 211, 111 (1984).

24 M. Takata, B. Umeda, E. Nishibori, M. Sakata, Y. Saitot, M. Ohno, and H. Shinohara, Nature (London) 377, 46 (1995).

25 S. W. Lovesey, Theory of Neutron Scattering from Condensed Matter, (Oxford University Press, 1984), Vol. 1. 